\title{
Molecular gas in galaxies: changing conditions from disks to starbursts
}

\author{
Christine D. Wilson \\ Department of Physics and Astronomy, McMaster University, \\ Hamilton, Ontario, Canada L8S 4M1 \\ email: wilsoncd@mcmaster.ca
}

\begin{abstract}
In understanding galaxy evolution, one central question is how star formation is regulated in galaxies. Changes in star formation rates are likely tied to changes in the interstellar medium, particularly the molecular gas which is the fuel for star formation. I will discuss our recent results which use data from the Herschel Space Observatory, the Atacama Large Millimeter/submillimeter Array, and other telescopes to determine the typical density, temperature, and surface density of the molecular gas in various nearby galaxies. Comparing the properties of molecular gas in starburst and other active galaxies with more quiescent spiral disks provides some clues as to how changes in the physical state of the gas, such as mean density, can lead to enhanced star formation rates.
\end{abstract}

Keywords. ISM: molecules, galaxies: spiral, galaxies: starburst

\section{Introduction}

The interstellar medium plays an important role in galaxies, providing both the immediate fuel for star formation (the molecular gas) and a larger reservoir for future star formation (the atomic gas). In the local Universe the interstellar medium accounts for a relatively small fraction of the total baryonic mass in galaxies. However, it is in nearby galaxies that radio and far-infrared telescopes have sufficient resolution to measure the gas and star-formation properties on the kiloparsec scales that are necessary for testing many models of star formation. The hope is that the physical processes uncovered in the local universe can then be applied to galaxies at high redshift, where the interstellar medium accounts for a much larger fraction of the mass in galaxies (e.g. Tacconi et al. 2013).

One interesting question concerns whether and how the molecular gas content of galaxies is affected by the broader environment in which both the gas and the galaxies live. Particularly in galaxy clusters, the effects of processes such as ram-pressure stripping and galaxy harassment can be seen in the distribution of the atomic gas; which processes affect the molecular gas and its rate of star formation is less well understood. In addition, the effects of local environment (e.g. arm versus interarm regions) inside spiral galaxies remains an area of interest.

A second interesting question concerns how the molecular gas in disk galaxies, where the star formation is proceeding at a steady rate in a kind of equilibrium, differs from that in starburst galaxies and mergers, where the huge rate of star formation limits the duration of this phase to perhaps a few hundred Myr. Given that many spectacular starburst galaxies are formed from the interaction of two disk galaxies, it is particularly interesting now to try to track the change in the molecular gas properties as the merger progresses, by studying a number of galaxies spread along the merger sequence. 


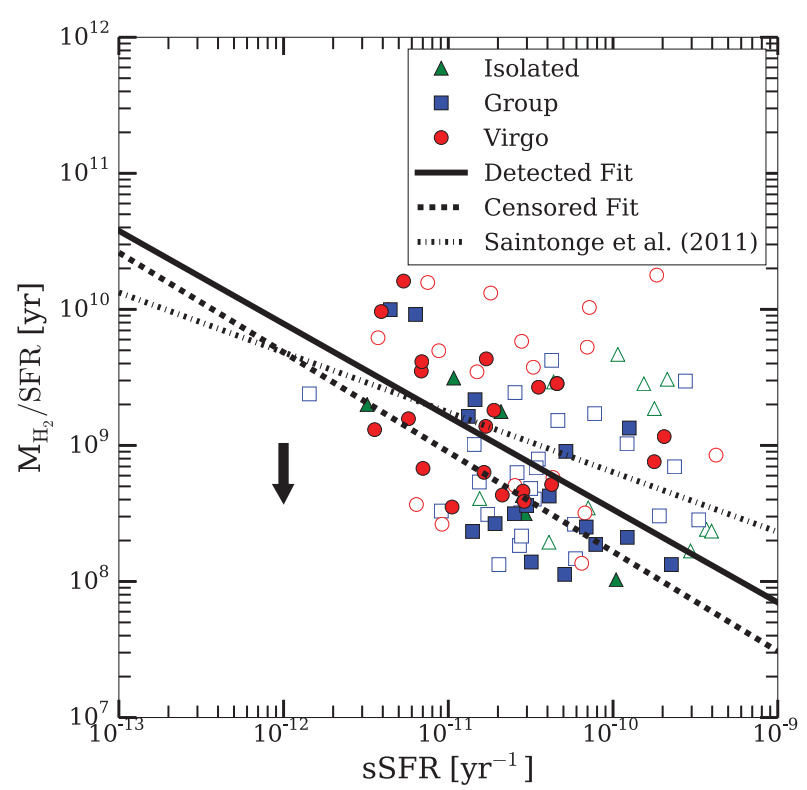

Figure 1. Molecular gas depletion time as a function of the specific star formation rate for spiral galaxies from the NGLS. The different symbols identify Virgo, group, and isolated spiral galaxies. Filled symbols are detections while open symbols are upper limits (the direction of the limits is indicated by the large arrow). The solid line is a linear fit to the detections while the dashed line is a fit including the upper limits (see Mok et al. 2016 for details). A similar fit from Saintonge et al. (2011) is shown as the dot-dashed line. Figure from Mok et al. 2016. (A color version of this figure is available online.)

In this contribution, I give an overview of some of the recent work that focuses on these two questions. Section 2 looks at the properties of nearby spiral galaxies, while Section 3 looks in detail at the particularly well-studied grand-design spiral, M51. Section 4 presents some new results for the Antennae, the closest merger between two large spirals, while Section 5 gives an overview of efforts to trace molecular gas properties along the merger sequence.

\section{How uniform are molecular gas properties in spiral galaxies?}

To first order, we may be tempted to view spiral galaxies in the local universe as a relatively uniform population in terms of their gas and star formation properties . For example, work by Bigiel et al. (2011) and others has shown that the star formation surface density correlates nearly linearly with the $\mathrm{H}_{2}$ surface density when averaged over kiloparsec scales in nearby spiral galaxies. However, evidence for changes in the CO-to- $\mathrm{H}_{2}$ conversion factor in galaxy centers as well as variations in the dust-to-gas mass ratio with metallicity suggest environmental dependencies lurk even inside this relatively uniform sample (Sandstrom et al. 2013).

One of the interesting questions that we can try to answer is whether and how the large-scale environment affects the molecular gas content of spiral galaxies. It has been clear for many years that the amount and distribution of atomic gas in galaxies can be strongly affected by environment, with many spirals showing a deficiency of atomic gas in cluster environments (e.g. Haynes, Giovanelli, \& Chincarini 1984, Solanes et al. 2001). However, Koopmann et al. (2006) showed that the star formation properties of galaxies are not as strongly affected by the cluster environment as is the atomic gas. Attempts to 
determine the effect of molecular gas on environment (Kenney \& Young 1989; Fumagalli \& Gavazzi 2008) have been hampered by a lack of a non-cluster sample of galaxies to use as a control sample. More recent studies of Virgo spirals include work by Cortese et al. (2012) on the dust scaling relations and Boselli et al. (2014) on the cold gas properties.

Mok et al. (2016 and this volume) have used the JCMT Nearby Galaxies Legacy Survey (NGLS, Wilson et al. 2012) to examine the effect of environment on the molecular gas content of spiral galaxies. The 98 spiral galaxies are classified as either Virgo Cluster members, members of other smaller groups of galaxies, or isolated galaxies. Galaxies in the Virgo cluster are found to have larger masses of molecular gas but relatively longer molecular gas depletion times relative to galaxies that reside in smaller groups. Interestingly, this suggests that the cluster environment is aiding in the conversion of atomic to molecular gas, but suppressing the rate at which that molecular gas turns into stars. Enhanced pressure from the intracluster medium might play a role in both these properties (see Usero et al. 2015 and Leroy, this volume).

The NGLS is unusual in being an HI-selected sample, in contrast to other surveys which are more commonly selected based on optical or near-infrared luminosity or on stellar mass. It is thus interesting to examine the correlations between various global properties to see if they are recovered in our sample. Fig. 1 shows a negative correlation between molecular gas depletion time and specific star formation efficiency $\left(\mathrm{SFR} / \mathrm{M}_{*}\right)$ that is consistent with results from other teams (Saintonge et al. 2011, Huang \& Kauffmann 2014, Boselli et al. 2014). This correlation implies that molecular gas is being converted more quickly into stars in those galaxies where the current star formation rate is high relative to the integrated past star formation rate. In effect, there is a link between the number of new stars being formed, the amount of gas available to form those stars, and the total number of stars that have been formed over the galaxies lifetime (see also Kannappan et al. 2013).

\section{M51: a case study of a disk galaxy}

As a gas-rich spiral seen relatively face-on, M51 (NGC 5194) has been a prime target for studying molecular gas in galaxies. Recent work includes Vlahakis et al. (2013), Schinnerer et al. (2013), and Pety et al. (2013). M51 was also one of 13 galaxies that were the target of the Very Nearby Galaxies Survey, a Guaranteed Time Key Program with the Herschel Space Observatory (PI: C. Wilson). Resolved modelling of the farinfrared spectral energy distribution using the Draine and Li (2007) models suggested somewhat elevated ultraviolet radiation fields compared to the solar neighborhood and a very low fraction of the dust being heated by young stars (Mentuch Cooper et al. 2012).

We have used data from the Herschel Space Observatory to approach the problem of determining the density and temperature of the molecular gas in M51 in two different ways. Parkin et al. (2013) used maps of far-infrared fine structure lines such as [CII], $[\mathrm{OI}]$, and $[\mathrm{NII}]$ combined with photon-dominated region (PDR) models from Kaufman et al. (1999) to constrain the molecular hydrogen density and the strength of the ultraviolet radiation field, $G_{o}$. Our analysis showed that the density and UV field strength were similar in the arm and interarm regions (Fig. 2). This result suggests that the average cloud properties are similar in the arm and interarm regions, despite having different gas and star formation rate surface densities on average. Both the average density and UV field strength were found to be higher towards the center of the galaxy.

We have also used observations of $\mathrm{CO}$ and $[\mathrm{CI}]$ at submillimeter wavelengths with the imaging Fourier Transform Spectrometer on Herschel to constrain these same physical conditions (Schirm 2015). We fit the CO lines with two components using the RADEX 

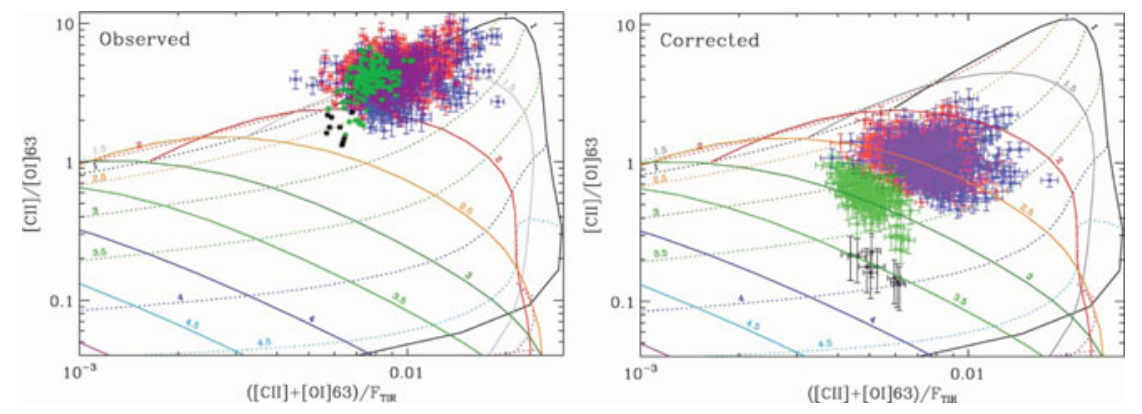

Figure 2. Fine-structure line data for M51 are shown overlaid on the PDR model grid from Kaufman et al. (1999). Dotted lines show $\log n_{H_{2}}$ and solid lines show $\log G_{o}$. Black, green, red, and blue pixels show data from the nucleus, center, arm, and interarm regions, respectively. The left panel shows observed quantities while the right panel shows the data after removal of the ionized gas contribution to [CII] and correction of [OI] for optical depth effects. Figure from Parkin et al. (2013). (A color version of this figure is available online.)

radiative transfer model (van der Tak et al. 2007) combined with a Bayesian likelihood code (Kamenetzky et al. 2011) to determine the density and temperature of the gas. These results are then compared to the PDR models from Hollenbach et al. (2012) to constrain the density and UV field strength of the molecular gas. For the cooler, lower density component in M51 which dominates the total mass in the models, we find similar average densities as in Parkin et al. (2013) but significantly lower UV field strength ( $\log G_{o} \leqslant 2$ compared to $\log G_{o} \sim 3$ ). Possible reasons for the discrepancy include the use of different PDR models in the two analyses as well as the addition of a constraint due to the CO line to far-infrared luminosity ratio in the analysis of Schirm (2015).

\section{The Antennae: a case study of a galaxy merger}

The merger system NGC 4038/9 known as "the Antennae" represents the closest example of a major merger between two gas-rich spiral galaxies. As such, it has been extensively studied at many wavelengths. It contains perhaps the largest collection of young massive star clusters of any single nearby galaxy (Whitmore et al. 1999) as well as a collection of super-giant molecular clouds (Wilson et al. 2003). However, its total gas content and global star formation rate are each within a factor of a few of similar properties of M51. Thus, a comparison of the Antennae with M51 may serve to highlight the similarities and differences between these two systems.

Schirm et al. (2014) carried out an analysis of the submillimeter CO and [CI] lines of the Antennae to determine the gas density and temperature. The method used was similar to that described above for M51. The two-component analysis of the molecular gas revealed that only about $0.3 \%$ of the molecular gas was in a warm, high-pressure phase (Fig. 3). This mass fraction is similar to what Schirm (2015) found for M51, but is substantially lower than the warm mass fraction of 10\% seen in M82 (Kamenetzky et al. 2012) and Arp 220 (Rangwala et al. 2011).

Recently, we have used the Atacama Large Millimeter/submillimeter Array (ALMA) to make high angular resolution maps of the Antennae in the dense gas tracers $\mathrm{HCN}, \mathrm{HCO}^{+}$, and HCN (Schirm et al. 2016). We see significant variations in the strengths of these lines relative to each other and also relative to $\mathrm{CO}$. In particular, $\mathrm{HCN}$ appears significantly enhanced relative to $\mathrm{CO}$ in the nuclear regions of the two galaxies that make up the 

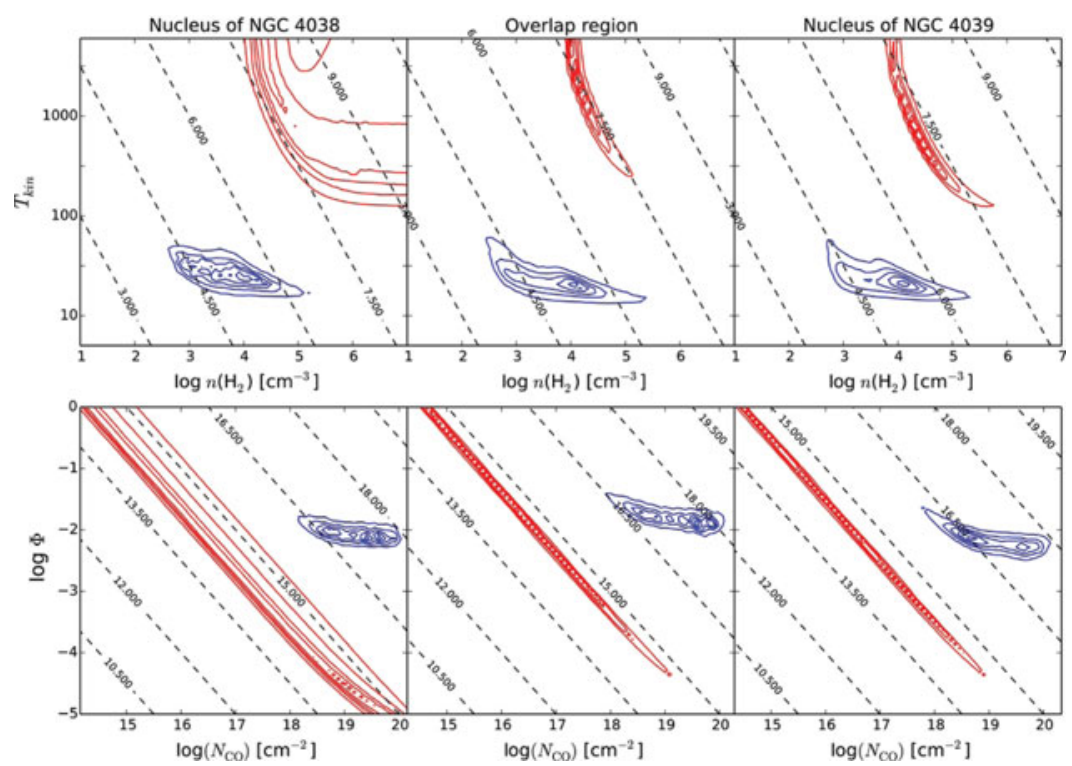

Figure 3. Contour probability plots from the Bayesian analysis of the $\mathrm{CO}$ and $[\mathrm{CI}]$ lines for three positions in the Antennae. The warm solution is shown as red contours and the cold solution as the blue contours. The top row plots kinetic temperature and density while the bottom row plots the filling factor and column density of the $\mathrm{H}_{2}$ gas. The dashed diagonal lines in the top row indicate $\log$ (Presssure) units of $\mathrm{Kcm}^{2}$; the dashed lines in the bottom row indicate the log of the average CO column density in units of $\mathrm{cm}^{-2}$. Figure from Schirm et al. (2014). (A color version of this figure is available online.)

Antennae system. These enhanced line ratios may trace increase gas pressure driven by the stronger potential of the stellar bulge in these regions (cf. Helfer \& Blitz 1993).

\section{Gas properties along the merger sequence}

As the closest ultraluminous infrared galaxy, Arp 220 presents probably the most extreme environment in terms of its star formation and gas properties in the local universe. We observed Arp 220 with the Herschel Space Observatory (Rangwala et al. 2011), revealing a submillimeter spectrum rich with emission and absorption lines of many molecules. A two-component analysis of the CO emission lines showed that $10 \%$ of the molecular gas mass was contained in an extremely warm $(T=1350 \mathrm{~K})$ component, while both the warm and the cooler components showed moderate $\mathrm{H}_{2}$ densities of $\log n \sim 3$. High resolution follow-up of the $\mathrm{CO} \mathrm{J}=6-5$ emission with ALMA to trace the morphology of the hot component revealed a very complex structure, with both red and blue-shifted absorption line and high optical depth towards each of the two nuclei (Rangwala et al. 2015 and this volume).

A central question in understanding the changes that take place in the star formation rates during galaxy interactions in mergers is determining how the physical properties of the molecular gas evolve as the merger progresses. Sliwa et al. (2012, 2013, 2014, 2015) have used high-resolution $\mathrm{CO}$ and ${ }^{13} \mathrm{CO}$ data for a small sample of merging galaxies from the sample of Wilson et al. (2008) to measure the physical conditions (density, temperature) and other properties such as ${ }^{13} \mathrm{CO}$ abundance and CO-to- $\mathrm{H}_{2}$ conversion factor in five ystems ranging from early to late phase mergers (Arp 55 (Fig. 4), Arp 299, NGC 1614, NGC 2623, and VV 114). This work has revealed interesting changes in the 


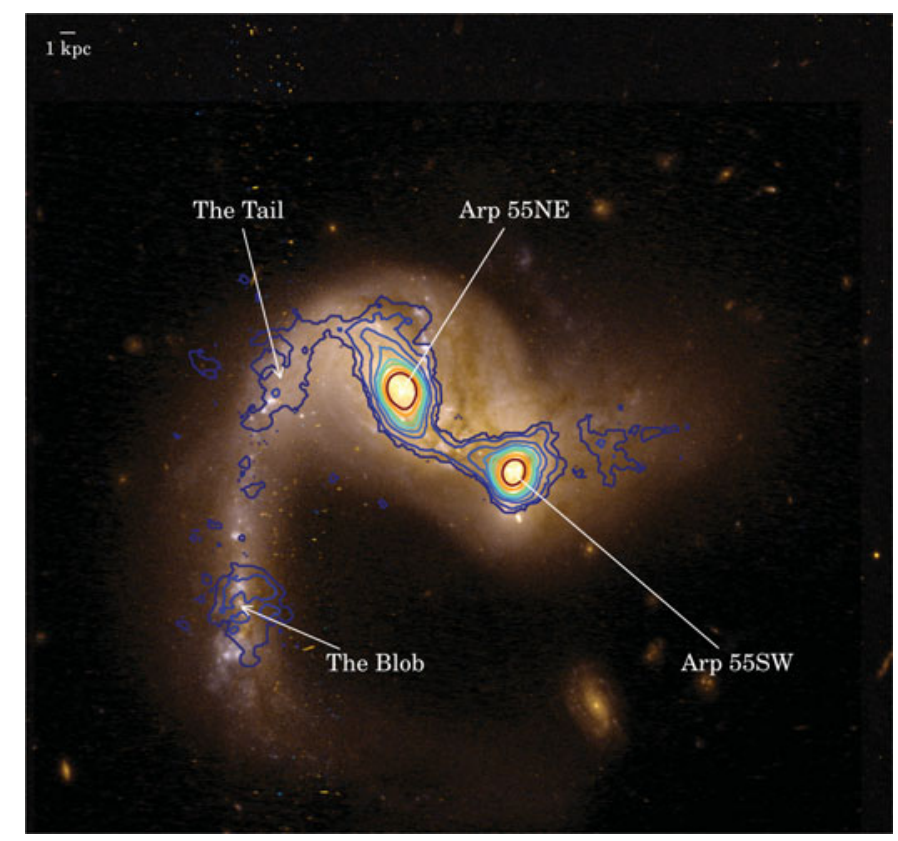

Figure 4. CO J=1-0 map from CARMA of the early-phase merger Arp 55 overlaid on an HST image. Figure from Sliwa (2015). (A color version of this figure is available online.)

mean molecular gas properties (see also Sliwa et al., this volume). Our analysis reveals that early and intermediate stage mergers typically have molecular gas that is colder and denser than that found in the late-type mergers (VV114, NGC2623). The data for Arp 220 (late) and the Antennae (early-intermediate) from Rangwala et al. (2011) and Schirm et al. (2014) conform to this trend as well.

High density gas is seen in Arp 220 (Wilson et al. 2014, Scoville et al. 2015) and in the Antennae (Schirm et al. 2016) in high resolution images from ALMA. However, our analysis suggests that the gas which is producing most of the CO emission has a substantially lower density than the gas found near the two nuclei in Arp 220 and in the Antennae. Given the complicated optical depth effects traced in the CO J=6-5 images of Arp 220 (Rangwala et al. 2015), this high density component may be nearly hidden from view in global analyses.

This result of lower density gas in advanced mergers is rather unexpected given the canonical picture of gas falling into the central regions as the merger progresses; in this picture, we would have expected higher density gas in the late-type mergers. One possible explanation is that the increasing feedback from stellar winds and supernova is beginning to dissipate the molecular gas in the most advanced systems, which could result in a lower average volume density. Recent observations showing evidence for massive molecular winds (e.g. Sturm et al. 2011) provide some support for this interpretation.

\section{Conclusions}

Data from new telescopes such as Herschel and ALMA combined with large surveys from single dish telescopes such as IRAM and the JCMT are opening up new possibilities for studying the link between star formation and the molecular interstellar medium in galaxies in the nearby universe. We are beginning to be able to answer the question of how environment affects the star formation process, be it inside a single galaxy comparing arm 
and inter-arm regions or radial trends, or larger external factors such as group or cluster environment, or the extreme cases of galaxy mergers. Initial surveys on the effect of cluster environment on the molecular gas in spirals (Mok et al. 2016) should soon be extended to other nearby clusters such as Fornax and Coma. Similarly, expanding the sample of mergers with well determined molecular gas properties beyond the seven systems studied so far (Sliwa 2015) will allow us to map out the evolution of the interstellar medium during galaxy mergers. Both directions are important for providing insights into evolutionary processes at work in the early universe.

\section{References}

Bigiel, F., Leroy, A. K., Walter, F., et al. 2011, AJ, 730, L13

Boselli, A., Cortese, L., Boquien, M., Boissier, S., Catinella, B., Lagos, C., \& Saintonge, A. 2014, $A \mathscr{E} A, 564, \mathrm{~A} 66$

Cortese, L., Ciesla, L., Boselli, A., et al. 2012, A\& A, 540, A52

Draine, B. T. \& Li, A. 2007, ApJ, 657, 810

Fumagalli, M. \& Gavazzi, G. 2008, A\&\&A, 490, 571

Haynes, M. P., Giovanelli, R., \& Chincarini, G. L. 1984, ARAA, 22, 445

Helfer, T. T. \& Blitz, L. 1993 ApJ, 419, 86

Hollenbach, D., Kaufman, M. J., Neufeld, D., Wolvire, M., \& Goicoechea, J. R. 2012, ApJ, 754, 105

Huang, M.-L. \& Kauffmann, G. 2014, MNRAS, 443, 1329

Kamenetzky, J., Glenn, J., Maloney, P. R.. et al. 2011, ApJ, 731, 83

Kamenetzky, J., Glenn, J., Rangwala, N. et al. 2012, ApJ, 753, 70

Kannappan, S. J., Stark, D. V., Eckert, K. D., et al. 2013, ApJ, 777, 42

Kaufman, M. J., Wolfire, M. G., Hollenback, D. J., \& Luhmn, M. L. 1999, ApJ, 527, 795

Kenney, J. D. P. \& Young, J. S. 1989, ApJ, 334, 171

Koopmann, R. A., Haynes, M. P., \& Catinella, B. 2006, AJ, 131, 716

Mentuch Cooper, E., Wilson, C. D., Foyle, K. et al. 2012, ApJ, 755, 165

Mok, A., Wilson, C. D., Golding, J. et al. 2016, MNRAS, submitted

Parkin, T. J., Wilson, C. D., Schirm, M. R. P., et al. 2013, ApJ, 776, 65

Pety, J., Schinnerer, E., Leroy, A. K. et al. 2013, ApJ, 779, 43

Rangwala, N., Maloney, P. R., Glenn, J. et al. 2011, ApJ, 743, 94

Rangwala, N., Maloney, P., Wilson, C. D., Glenn, J., Kamenetzky, J., \& Spinoglio, L. 2015, ApJ, 806, 17

Saintonge, A., Kauffmann, G., Wang, J., et al. 2011, MNRAS, 415, 61

Sandstrom, K. M., Leroy, A. K., Walter, F., et al. 2013, ApJ, 777, 5

Schinnerer, E., Meidt, S. E., Pety, J. et al. 2013, ApJ, 779, 42

Schirm, M. R. P., Parkin, T. J., Wilson, C. D., et al. 2014, ApJ, 781, 101

Schirm, M. R. P. 2015, Ph.D. thesis, McMaster University

Schirm, M. R. P., Wilson, C. D., Madden, S. C., \& Clemens, D. L. 2016, ApJ, submitted

Scoville, N., Sheth, K., Walter, F., et al. 2015, ApJ, 800, 70

Sliwa, K., Wilson, C. D., Petitpas, G. R. et al. 2012, ApJ, 753, 46

Sliwa, K., Wilson, C. D., Krips, M. et al. 2013, ApJ, 796, L15

Sliwa, K., Wilson, C. D., Iono, D., Peck, A., \& Matsushita, S. 2014, ApJ, 753, 46

Sliwa, K. 2015, Ph.D. thesis, McMaster University

Solanes, J. M., Manrique, A., García-Gómez, C., González-Casado, G., Giovanelli, R., \& Haynes, M. P. 2001, ApJ, 548, 97

Sturm, E., González-Alfonso, E., Veilleux, S., et al. 2011, ApJ, 733, L16

Tacconi, L., Neri, R., Genzel, R., et al. 2013, ApJ, 768, 74

Usero, A., Leroy, A. K., Walter, F., et al. 2015, AJ, 150, 115

van der Tak, F. F. S., Black, J. H., Schier, F. L., Jansen, D. J., \& van Dishoeck, E. F. 2007, $A \mathscr{E} A, 468,627$

Vlahakis, C., van der Werf, P., Israel, F. P., \& Tilanus, R. P. J. 2013, MNRAS, 433, 1837 
Whitmore, B. C., Zhang, Q., Leitherer, C., Fall, S. M., Schweizer, F., \& Miller, B. W. 1999, AJ, 118,1551

Wilson, C. D., Scoville, N., Madden, S. C., \& Charmandaris, V. 2003, ApJ, 599, 1049

Wilson, C. D., Petitpas, G. R., Iono, D. et al. 2008, ApJS, 178, 189

Wilson, C. D., Warren, B. E., Israel, F., et al. 2012, MNRAS, 424, 3050

Wilson, C. D., Rangwala, N., Glenn, J., Maloney, P., Spinoglio, L., \& Pereira-Ssantaella, M. 2014, ApJ, 789, L36 